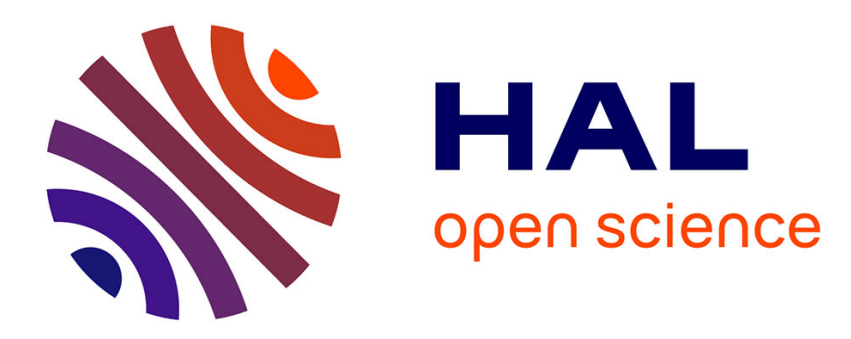

\title{
Anomalous Multiphoton Photoelectric Effect in Ultrashort Time Scales
}

J Kupersztych, M Raynaud

\section{To cite this version:}

J Kupersztych, M Raynaud. Anomalous Multiphoton Photoelectric Effect in Ultrashort Time Scales. Physical Review Letters, 2005, 95, pp.147401. 10.1103/PhysRevLett.95.147401 . hal-01327441

\section{HAL Id: hal-01327441 \\ https://hal.science/hal-01327441}

Submitted on 14 Jun 2016

HAL is a multi-disciplinary open access archive for the deposit and dissemination of scientific research documents, whether they are published or not. The documents may come from teaching and research institutions in France or abroad, or from public or private research centers.
L'archive ouverte pluridisciplinaire HAL, est destinée au dépôt et à la diffusion de documents scientifiques de niveau recherche, publiés ou non, émanant des établissements d'enseignement et de recherche français ou étrangers, des laboratoires publics ou privés. 


\title{
Anomalous Multiphoton Photoelectric Effect in Ultrashort Time Scales
}

\author{
J. Kupersztych and M. Raynaud \\ DSM/DRECAM/SPCSI, CEA/Saclay, 91191 Gif-sur-Yvette, France
}

(Received 17 December 2004; published 29 September 2005)

\begin{abstract}
In a multiphoton photoelectric process, an electron needs to absorb a given number of photons to escape the surface of a metal. It is shown for the first time that this number is not a constant depending only on the characteristics of the metal and light, but varies with the interaction duration in ultrashort time scales. The phenomenon occurs when electromagnetic energy is transferred, via ultrafast excitation of electron collective modes, to conduction electrons in a duration less than the electron energy damping time. It manifests itself through a dramatic increase of electron production.
\end{abstract}

DOI: 10.1103/PhysRevLett.95.147401

PACS numbers: 78.70. $-\mathrm{g}$, 73.20.Mf, 79.60. $-\mathrm{i}$

A basic hypothesis of the photoelectric process is that the photoemissive properties of matter remain unaltered during the interaction with light. Light-metal coupling is tacitly assumed as a perturbation of the electron population that remains in equilibrium during the interaction. Now, it has recently been shown that transient nonequilibrium electron states can exist in ultrashort time scales, in particular, when electromagnetic energy is transferred from a laser pulse to conduction electrons in a lapse of time shorter than the electron-phonon energy transfer duration [1-4]. In this Letter, we address the basic question of whether the photoemissive properties of a metal can be modified through ultrafast energy transfer and nonequilibrium electron heating.

In a metallic electron gas, transient density disturbances can result in electron collective oscillation modes in the volume and near the surface. Under certain conditions, these so-called surface plasmon (polariton) modes can be excited by light $[5,6]$. In the case of thin metal films, the surface plasmon modes on the two surfaces can be coupled [7-9] and energy can be transferred from one surface plasmon mode to the other [10]. Collective electron oscillations can exist as well at the interface [11] between two perfect metals due to symmetry breaking at the metalmetal interface. Furthermore, interface and surface plasmon modes can be coupled [12] in a bilayer metal system made of a metal $M_{1}$ (of electron density $n_{1}$ ) covered by a thin metallic layer $M_{2}$ (of electron density $n_{2}<n_{1}$ ). If the overlayer metal $M_{2}$ is thin enough, the field of the surface plasmon can tunnel through the $M_{2}$ bulk and excite electron density fluctuations at the interface between the two metals (see Fig. 1). If the metal overlayer is too thick, the field of the surface plasmon must tunnel through too large a distance to excite the density fluctuations between the two metals. Conversely, if it is too thin, the surface plasmon amplitude is damped because of increasing coupling between the two opposite faces of the overlayer. There exists therefore an optimum thickness of the overlayer for which the amplitude of the induced interface plasmon is maximum.
An interesting consequence of the interface or surface plasmon coupling effect is that the electron population in the metal overlayer can be in transient nonequilibrium energy states through ultrafast energy transfer from the coupled interface and surface plasmons. Actually, the conduction electrons near the surface and the metal-metal interface experience an effective nonlinear low-frequency force, the so-called ponderomotive force $[13,14]$, resulting from the strongly inhomogeneous high-frequency field of the plasmons, and are accelerated toward regions of decreasing field amplitude. The ponderomotive force plays the role of an applied electrostatic force that transfers electromagnetic energy in a coherent way to an electron population, in contrast with stochastic energy transfer via thermal heating. The maximum energy that can be transferred to a free electron with initial energy $\mathcal{E}_{0}$ through ponderomotive acceleration in a strong oscillating electric

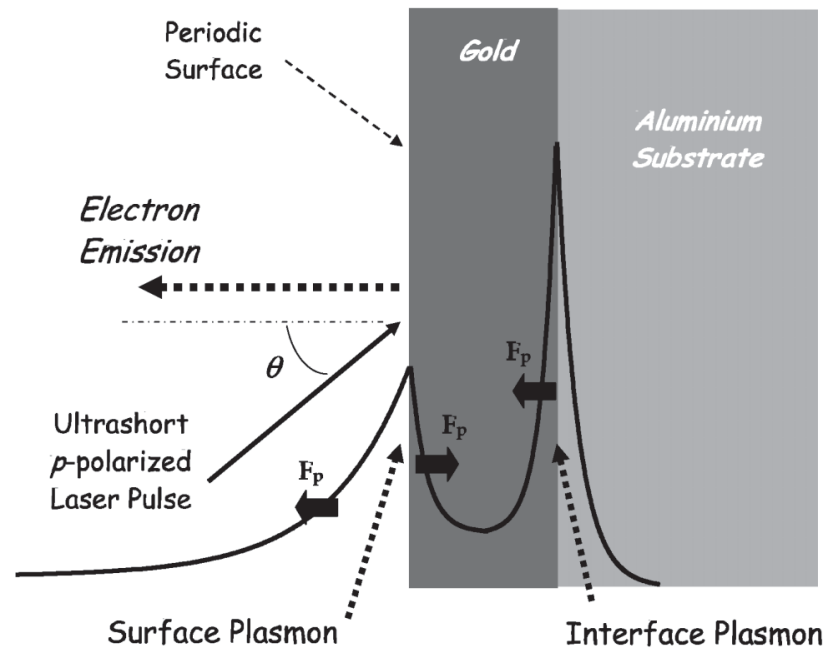

FIG. 1. Schematic representation of the resonant laser excitation of the interface or surface plasmon coupled system. For ultrashort laser pulse durations, energy is transferred through the ponderomotive forces $F_{p}$ corresponding to the interface and plasmon fields to the conduction electron population of the overlayer. 
field $E$ of angular frequency $\omega$ is $\mathcal{E}_{\max }=3 U_{p}+$ $2^{3 / 2}\left(\mathcal{E}_{0} U_{p}\right)^{1 / 2}$, where $U_{p}=e^{2} E^{2} / 4 m \omega^{2}$ denotes the ponderomotive potential [15]. The part played by the ponderomotive force of surface plasmons on the energy spectra of the electrons emitted in the vacuum has been previously identified [15]. In this context, it has been shown that surface plasmons with ponderomotive potentials of several $\mathrm{eV}$ can be resonantly excited by low-intensity short laser pulses.

Similarly, electromagnetic energy can be coherently transferred to conduction electrons in the metal overlayer by the ponderomotive forces of the surface and interface plasmons. As a consequence, those electrons in the skin depth that have experienced ponderomotive acceleration need less energy to overcome the potential barrier at the metal surface. This effect could therefore be detected by measuring a lower threshold in linear photoemission. However, as surface plasmon excitation requires light in the infrared or visible range, electron emission cannot occur via a linear process but through a multiphoton photoelectric effect [16-18]. In this process, release of an electron with energy $\mathcal{E}$ from the metal surface requires the absorption of a minimum number $N$ of photons, as described by the (generalized) Einstein photoelectric equation: $\mathcal{E}=N \hbar \omega-W_{M}$ (where $W_{M}$ denotes the metal work function and $\omega$ the angular frequency of light). The photoelectron current density $J$ which depends on the light intensity $I$ through an $N$-power law, $J \propto \sigma_{N} I^{N}$, where $\sigma_{N}$ denotes the cross section of the $N$-photon process, is thus characterized in log-log coordinates by a linear curve of slope $N$. Hence, ultrafast energy transfer from the coupled interface and surface plasmons can result in a reduction of the number of photons needed to be absorbed by the electrons to escape the metal surface, which can be experimentally detected by measuring a smaller integer value of the slope in the photoemission process.

In our experiment, resonant excitation of the interface or surface plasmon system was achieved by means of a $p$-polarized laser impinging onto a bilayer grating target with an incidence angle $\theta$ (see Fig. 1). We used a Ti:sapphire (unfocused) laser of wavelength $\lambda=800 \mathrm{~nm}$ delivering pulses, the duration of which could be varied from $50 \mathrm{fs}$ to $1.2 \mathrm{ps}$ with a repetition rate of $20 \mathrm{~Hz}$. Stability of laser pulse durations and intensities were measured to within $10 \%$. To avoid any thermal effect that could interfere with the photoemission process $[16,17]$, we employed laser pulses of low intensities $\left(I \leq 1 \mathrm{GW} / \mathrm{cm}^{2}\right)$. All recorded data were accumulated over 1000 laser shots. The bimetal gratings were obtained by vacuum deposition of a $200 \mathrm{~nm}$ thick Al film on a commercial grating (of period $a=6.7 \mu \mathrm{m}$ ) and subsequent deposition of gold with various thicknesses by joule evaporation. Overlayer thicknesses were measured by surface profilometry and uniformity was checked by optical measurements. All the emitted electrons were collected through a grid on an electron multiplier (microchannel plates). The measured resonance angle corresponding to the optimum electron emission was $\theta_{\text {res }} \simeq 65^{\circ} \pm 0.2^{\circ}$ (see Fig. 2). The angular dispersion was remarkably sharp (less than $2^{\circ}$ ) [19], which showed that extreme accuracy in the handling of the various samples is a sine qua non condition for the detection of electron emission and for a correct determination of its dependence versus laser intensity [20].

As the work function of polycrystalline gold is $W_{\mathrm{Au}}=$ $5.1 \mathrm{eV}$ [21], emission of an electron a priori requires the absorption of 4 photons of energy $\hbar \omega=1.55 \mathrm{eV}(\lambda=$ $800 \mathrm{~nm}$ ). Actually, the measured photoelectron currents versus laser intensity shown on Fig. 3 present a slope 4 for the sample of gold thickness $200 \mathrm{~nm}$ (for which the interface plasmon could not be excited). For the massive aluminum sample, the measured slope is 3 in agreement with the value of its work function $W_{\mathrm{Al}}=4.2 \mathrm{eV}$ [21]. Both samples present similar photoelectric currents. A remarkable result is that, for samples with a gold overlayer thickness of nearly $43 \mathrm{~nm}$, the slope becomes equal to 3 while the electron current increases dramatically. This slope 3 is also observed for those samples of thickness 18 and $28 \mathrm{~nm}$ only after some threshold value of the laser intensity. Hence, the generalized photoelectric equation with a normal value of the gold work function $\left(W_{\mathrm{Au}}=\right.$ $5.1 \mathrm{eV}$ ) is apparently no longer satisfied. Noting the value of the penetration depth $(\delta \simeq 36 \mathrm{~nm})$ of infrared light in gold, it can be concluded that the anomalous photoelectric effect occurs when the gold overlayer thickness is of the same order as the skin depth.

The photoelectric anomaly is a short-living transient effect that disappears for longer time scales because of

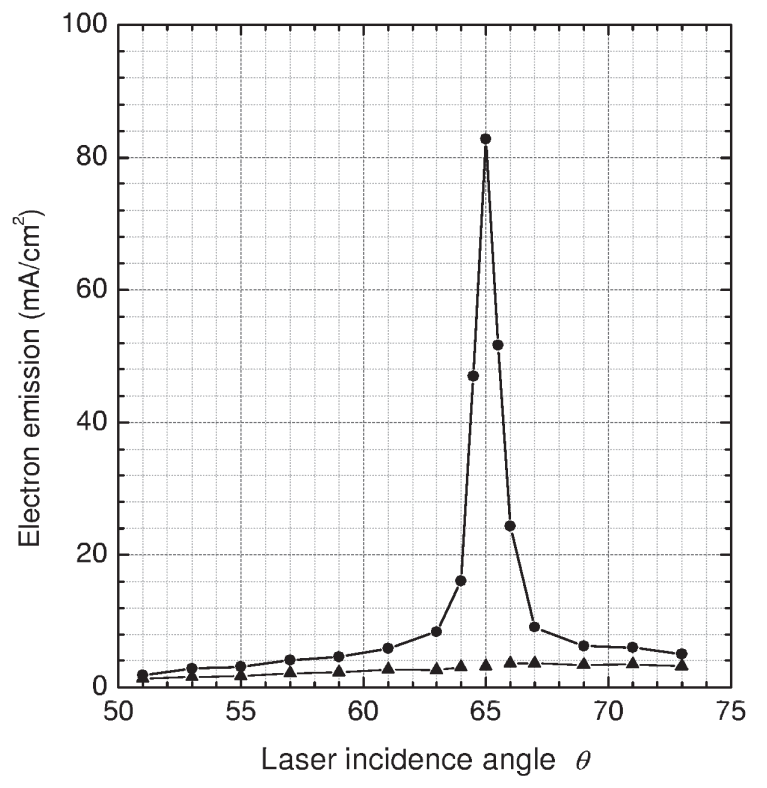

FIG. 2. Photoelectron emission versus incidence angle $\theta$ for $p$-polarized (O) and $s$-polarized (A) laser pulses (at $50 \mathrm{fs}$ and for $\left.I=1 \mathrm{GW} / \mathrm{cm}^{2}\right)$. 


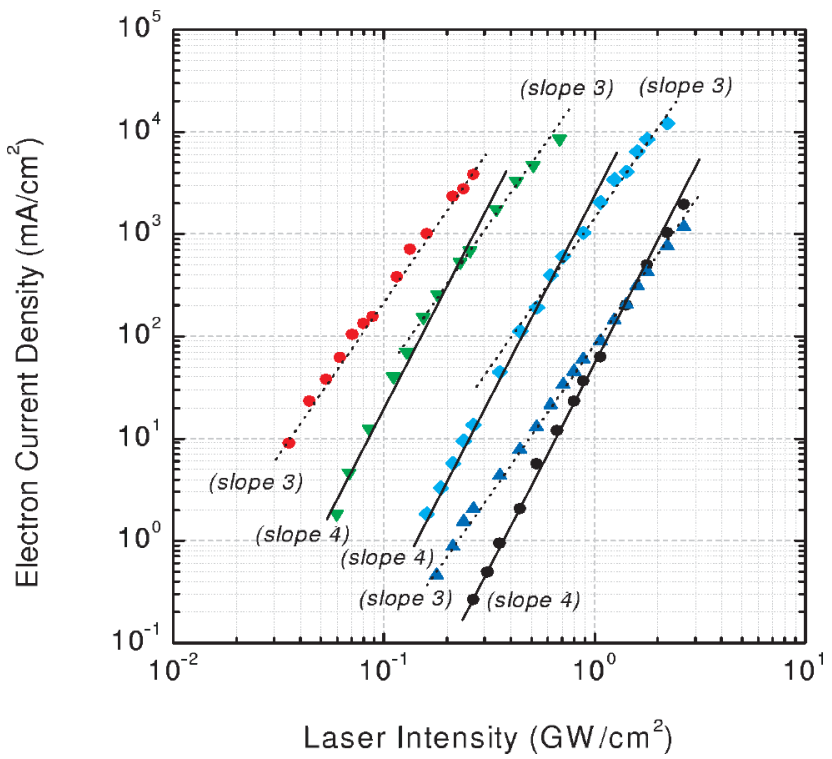

FIG. 3 (color). Photoelectron current versus laser intensity (pulse duration $50 \mathrm{fs}$ ) for several typical samples: ultrathick $(200 \mathrm{~nm})$ gold $(\boldsymbol{O})$, massive aluminum $(\boldsymbol{\Delta})$, and gold overlayers of thickness: $18 \mathrm{~nm}(\boldsymbol{\nabla}), 28 \mathrm{~nm}(\boldsymbol{\nabla})$, and $43 \mathrm{~nm}$ (red circles). For the optimum overlayer thickness $(43 \mathrm{~nm})$, the number of absorbed photons in the photoemission process turns out to 3 instead of 4 (as required by the generalized photoelectric equation). The same slope changes $(4 \rightarrow 3)$ occur for samples of thickness 18 and $28 \mathrm{~nm}$ at higher laser intensity (i.e., at higher plasmon amplitude).

coupling between the electron collective modes and the crystal lattice. Actually, as plasmon-phonon interactions come into play for lapses of time of the order of $1 \mathrm{ps}$ [22], the outbreak of resonance dissipation is expected to take place for laser pulse durations in the picosecond range. Transience of the photoelectric anomaly is clearly seen in Fig. 4. Thus, when the laser pulse duration is in the picosecond range (1.2 ps), the characteristic dependence of the photoelectric current versus laser intensity corresponding to the optimum sample turns back to the expected slope 4 while the "anomalous" slope 3 is still found for pulse lengths less than $400 \mathrm{fs}$. It is worth emphasizing that no measurable slope change was observed for the ultrathick $(200 \mathrm{~nm})$ gold or massive aluminum samples in this pulse range and in this low-intensity regime $\left(I<1 \mathrm{GW} / \mathrm{cm}^{2}\right)$ [23].

The relationship between the photoelectric anomaly and the amplitude of the plasmon resonance can also be drawn from the spectra of the photoelectrons. It has recently been shown [15] that the electron energy spectra are shifted toward higher energies as the laser pulse durations increase in the femtosecond regime. These shifts were interpreted as due to the acceleration experienced by the emitted electrons in the vacuum by the ponderomotive force of the surface plasmon field, the amplitude of which is growing during the laser pulse duration [15,24,25]. In the present

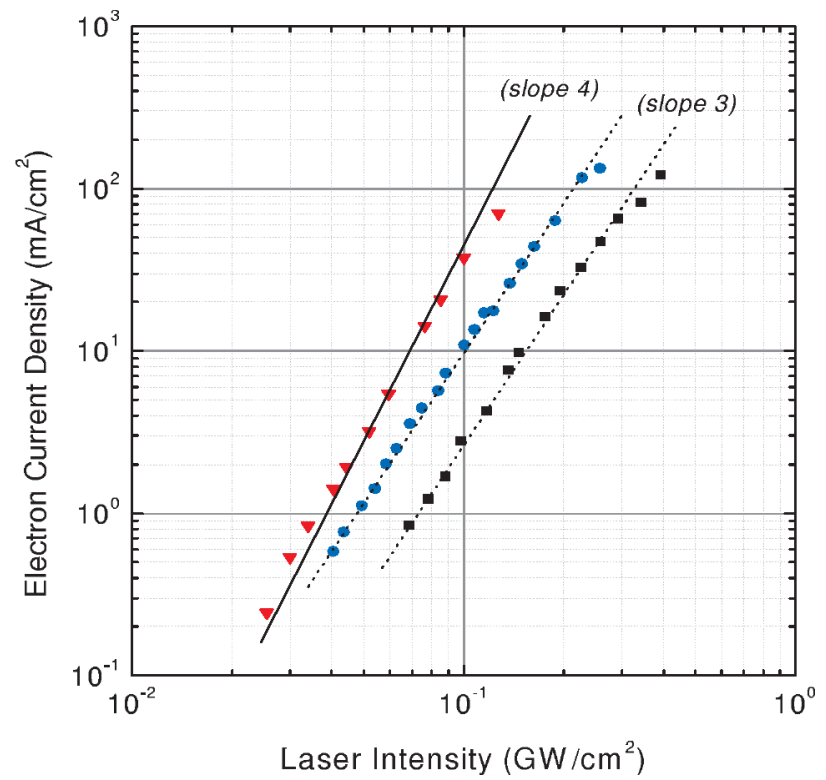

FIG. 4 (color). Photoelectron current versus laser pulse intensity for an optimum sample (gold overlayer thickness near $40 \mathrm{~nm}$ ), for pulse durations: $50 \mathrm{fs}(\boldsymbol{\square}), 400 \mathrm{fs}(\boldsymbol{)})$, and $1.2 \mathrm{ps}$ $(\boldsymbol{\nabla})$. In the femtosecond regime (50-400 fs), the photoelectric emission shows the absorption of an anomalous number of photons ( 3 instead of 4 ). In the picosecond range, the slope turns back $(3 \rightarrow 4)$ to the normal number of photons required by the generalized photoelectric equation.

case, electron emission was analyzed (at maximum of the resonance with a time-of-flight magnetically shielded spectrometer with energy resolution of $0.1 \mathrm{eV}$ ) for laser pulse durations ranging from $50 \mathrm{fs}$ to $1.6 \mathrm{ps}$. It now appears that, for laser pulse lengths larger than $800 \mathrm{fs}$, the electron

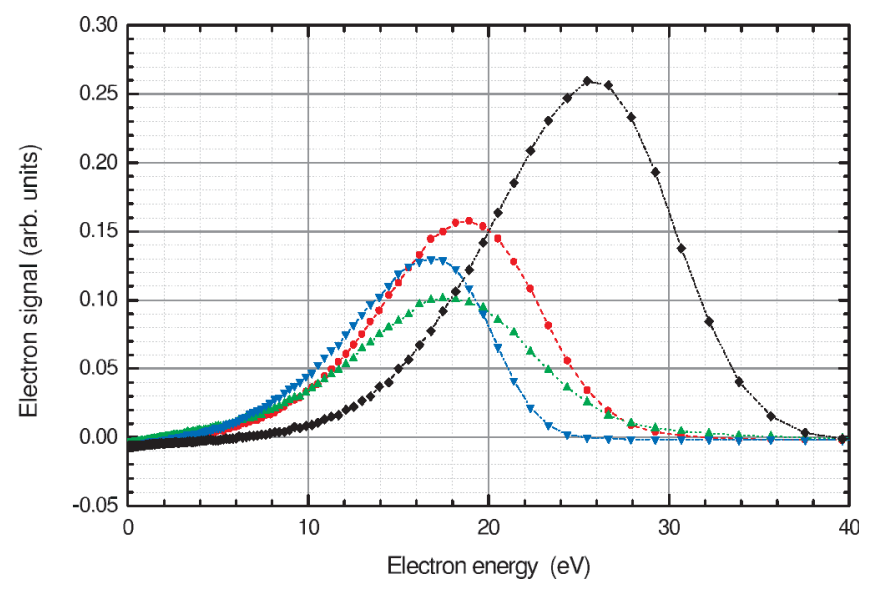

FIG. 5 (color). Photoelectron energy spectra of the ultrathick sample (i.e., without interface plasmon excitation) as a function of the laser pulse duration (for $I=1 \mathrm{GW} / \mathrm{cm}^{2}$ ): $400 \mathrm{fs}(\boldsymbol{\Delta})$, $800 \mathrm{fs}(\bullet), 1.2 \mathrm{ps}(\boldsymbol{\bullet}), 1.6 \mathrm{ps}(\boldsymbol{\nabla})$. In the femtosecond regime, the spectra shift toward higher energies [15,24], due to a growing of the surface plasmon amplitude. In the picosecond range, the spectra shift toward lower energies due to a decreasing of the plasmon amplitude in the long-pulse regime. 


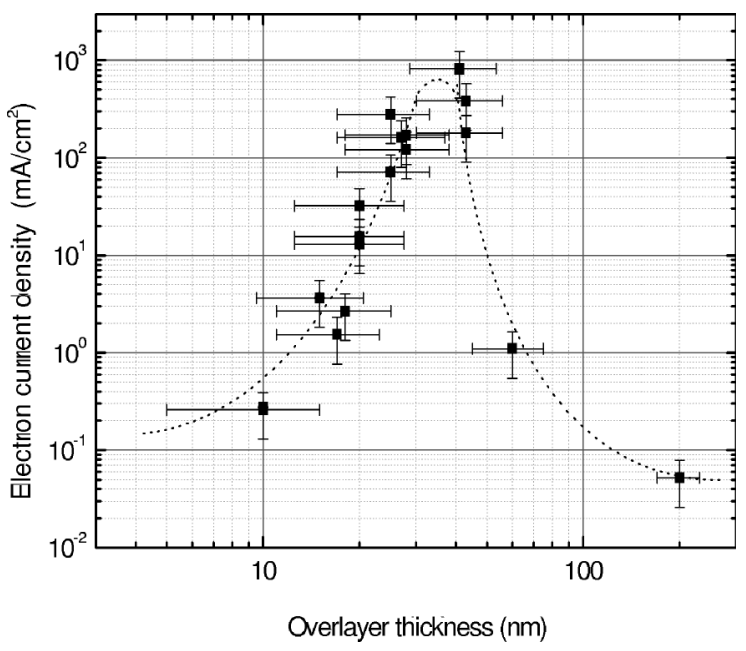

FIG. 6. Photoelectron current as a function of the gold overlayer thickness (at $50 \mathrm{fs}$ and for $I=0.3 \mathrm{GW} / \mathrm{cm}^{2}$ ). For a gold overlayer thickness of nearly $43 \mathrm{~nm}$ the photoelectron emission exhibits a dramatic enhancement compared to the ultrathick gold or massive aluminum samples.

spectra are then reversely shifted toward lower energies (see Fig. 5). It can thus be concluded that, in the picosecond range, the amplitude of the surface plasmon is damped through coupling between the electrons and the crystal lattice and the emitted photoelectrons are less accelerated. Correlatively, the multiphoton photoelectric emission corresponds to a return-to-equilibrium physical situation as confirmed by the characteristic slope 4 shown on Fig. 4.

Let us add that the anomalous multiphoton photoelectric effect described here can also have interesting practical applications for the photoemission efficiency. As, according to perturbation theory, the probability of an $N$-photon process decreases as the number $N$ of required photons increases, it can be expected that the photoelectron signal be enhanced when the optimum conditions for a photoelectric anomaly are met. As it is shown on Fig. 6, the effect is clearly significant. The amplitude of the photoemission signal exhibits an increase by a factor greater than $10^{4}$ compared to the ultrathick gold or massive aluminum samples, for a gold overlayer thickness of nearly $43 \mathrm{~nm}$, which is just in the range of gold overlayer thicknesses that yield the slope 3 for the photoelectron current. Thus, taking into account the enhancement of the photoelectron yield in surface-plasmon-assisted photoemission [18], the present method appears as an efficient tool for improving the photoelectron production by at least a factor of the order of $10^{6}$. This result makes the anomalous multiphoton photoelectric effect a valid candidate to be taken into consideration for the development of high-performance short-pulse electron sources.
We thank G. Lechevalier and F. Picard for their contribution at various stages of the experiments. The technical staff of the LUCA Saclay Laser Facility is also gratefully acknowledged.

[1] J. G. Fujimoto et al., Phys. Rev. Lett. 53, 1837 (1984).

[2] R. W. Schoenlein et al., Phys. Rev. Lett. 58, 1680 (1987).

[3] C. Suàrez, W. E. Bron, and T. Juhasz, Phys. Rev. Lett. 75, 4536 (1995).

[4] C. Guo, G. Rodriguez, and A. T. Taylor, Phys. Rev. Lett. 86, 1638 (2001).

[5] R. H. Ritchie et al., Phys. Rev. Lett. 21, 1530 (1968).

[6] H. Raether, Surface Plasmons on Smooth and Rough Surfaces and on Gratings (Springer-Verlag, Berlin, 1988).

[7] R. H. Ritchie and H.B. Eldridge, Phys. Rev. 126, 1935 (1962).

[8] E. N. Economou, Phys. Rev. 182, 539 (1969).

[9] G. P. Bryan-Brown, S. J. Elston, and J. R. Sambles, Opt. Commun. 82, 1 (1991).

[10] J. Seidel et al., Phys. Rev. B 69, 121405 (2004).

[11] E. A. Stern and R. A. Ferrel, Phys. Rev. 120, 130 (1960).

[12] J. Kupersztych and M. Raynaud, Solid State Commun. 106, 785 (1998); Phys. Rev. E 59, 4559 (1999).

[13] J. Kupersztych, Phys. Rev. Lett. 54, 1385 (1985).

[14] P. Agostini et al., Phys. Rev. A 36, 4111 (1987).

[15] J. Kupersztych, P. Monchicourt, and M. Raynaud, Phys. Rev. Lett. 86, 5180 (2001).

[16] J.H. Bechtel, W. Lee Smith, and N. Bloembergen, Phys. Rev. B 15, 4557 (1977).

[17] R. Yen, J. Liu, and N. Bloembergen, Opt. Commun. 35, 277 (1980).

[18] J. Hofmann and W. Steinmann, Phys. Status Solidi 30, K53 (1968); J. Endriz and W. Spicer, Phys. Rev. Lett. 24, 64 (1970); J. T. Stuckless and M. Moskovits, Phys. Rev. B 40, 9997 (1989); T. Tsang, T. Srivanasan-Rao, and J. Fisher, ibid. 43, 8870 (1991); V. Shalaev et al., ibid. 53, 11193 (1996).

[19] R. A. Watts, T. W. Preist, and J. R. Sambles, Phys. Rev. Lett. 79, 3978 (1997).

[20] In preliminary experiments performed in our laboratory [S. Bastiani et al., J. Phys. IV (France) 11, Pr7 (2001)], imperfect resonance conditions resulted in a much lower electron signal.

[21] A.H. Sommer, Photoemissive Materials (Krieger, Huntington, 1980).

[22] H.E. Elsayed-Ali et al., Phys. Rev. Lett. 58, 1212 (1987).

[23] Thermal contributions to electron production via thermionic emission would lead to an increase of the slopes (to noninteger values) (see, e.g., Ref. [17]).

[24] J. Kupersztych et al., J. Phys. B 21, L517 (1988).

[25] J. Zawadzka et al., Appl. Phys. Lett. 79, 2130 (2001); S. E. Irvine, A. Dechant, and A. Y. Elezzabi, Phys. Rev. Lett. 93, 184801 (2004). 